\title{
In vivo monitoring of adult neurogenesis in health and disease
}

\author{
Sebastien Couillard-Despres ${ }^{1}$, Ruth Vreys ${ }^{2}$, Ludwig Aigner ${ }^{1}$ and Annemie Van der Linden ${ }^{2 *}$ \\ Institute of Molecular Regenerative Medicine, Paracelsus Medical University, Salzburg, Austria \\ 2 Bio-Imaging Lab, Department of Biomedical Sciences, University of Antwerp, Antwerp, Belgium
}

\section{Edited by:}

Silvia De Marchis, University of Turin,

Italy

\section{Reviewed by:}

Jason Lerch, Toronto Center for

Phenogenomics, Canada

Maurice Curtis, Auckland University,

New Zealand

\section{*Correspondence:}

Annemie Van der Linden, Bio-Imaging Lab, University of Antwerp,

Universiteitsplein 1, B-2610 Antwerpen

(Wilrijk), Belgium.

e-mail: annemie.vanderlinden@ua. ac.be
Adult neurogenesis, i.e., the generation of new neurons in the adult brain, presents an enormous potential for regenerative therapies of the central nervous system. While 5-bromo-2'-deoxyuridine labeling and subsequent histology or immunohistochemistry for cell-type-specific markers is still the gold standard in studies of neurogenesis, novel techniques, and tools for in vivo imaging of neurogenesis have been recently developed and successfully applied. Here, we review the latest progress on these developments, in particular in the area of magnetic resonance imaging (MRI) and optical imaging. In vivo in situ labeling of neural progenitor cells (NPCs) with micron-sized iron oxide particles enables longitudinal visualization of endogenous progenitor cell migration by MRI. The possibility of genetic labeling for cellular MRI was demonstrated by using the iron storage protein ferritin as the MR reporter-gene. However, reliable and consistent results using ferritin imaging for monitoring endogenous progenitor cell migration have not yet been reported. In contrast, genetic labeling of NPCs with a fluorescent or bioluminescent reporter has led to the development of some powerful tools for in vivo imaging of neurogenesis. Here, two strategies, i.e., viral labeling of stem/progenitor cells and transgenic approaches, have been used. In addition, the use of specific promoters for neuronal progenitor cells such as doublecortin increases the neurogenesis-specificity of the labeling. Naturally, the ultimate challenge will be to develop neurogenesis imaging methods applicable in humans. Therefore, we certainly need to consider other modalities such as positron emission tomography and proton magnetic resonance spectroscopy ( $\left.{ }^{1} \mathrm{H}-\mathrm{MRS}\right)$, which have already been implemented for both animals and humans. Further improvements of sensitivity and neurogenesis-specificity are nevertheless required for all imaging techniques currently available.

Keywords: neurogenesis, MRI, MRS, PET, bioluminescence, fluorescence, optical imaging, MPIO

\section{INTRODUCTION}

The existence of adult neurogenesis in the mammalian brain has been demonstrated almost half of a century ago (Altman and Das, 1965), nevertheless, broad attention to this process only arose after the discovery of pools of neural stem-like cells residing in discrete brain regions (Reynolds and Weiss, 1992). In most adult mammals, proliferating neural stem cells can be detected in two neurogenic niches, the subventricular zone (SVZ) of the lateral ventricle wall and the subgranular zone (SGZ) of the hippocampal dentate gyrus (see Ma et al., 2009 for a recent review). The SVZ constitutes the most active site of neurogenesis (Conover and Allen, 2002). Young neural progenitor cells (NPCs), also known as neuroblasts, are generated throughout the entire SVZ and subsequently migrate along the rostral migratory stream (RMS) into the olfactory bulb $(\mathrm{OB})$ where they differentiate into new interneurons (Lois and Alvarez-Buylla, 1994). In the SGZ, neurogenesis occurs at a lower rate compared to the SVZ (Altman and Das, 1965; Cameron et al., 1993) and, in contrast to the SVZ-derived neuroblasts, new neurons of the SGZ migrate only short distances into the overlying granule cell layer (Stanfield and Trice, 1988; Markakis and Gage, 1999). Great hope followed the discovery that active populations of neural stem cells also generate new neurons within the adult human brain (Eriksson et al., 1998). Endogenous stem cell-based therapy for neuroregeneration after brain injury appeared suddenly nearer.
Clinical and preclinical studies have shown that adult neurogenesis is altered in response to brain insults with neuronal death. For example, ischemic lesions stimulate cell proliferation in the SVZ and the SGZ (Parent et al., 1997; Liu et al., 1998; Jin et al., 2001; Zhang et al., 2001; Arvidsson et al., 2002). Furthermore, it has been reported that new neurons migrate to non-neurogenic regions lining the ischemic lesion sites (Magavi et al., 2000; Arvidsson et al., 2002; Parent et al., 2002).

Postmortem studies on patients suffering from Huntington's disease (HD) demonstrated increased cell proliferation in the neurogenic regions (Curtis et al., 2003, 2005). Nonetheless, failure of the reparative process has been observed in animal models of $\mathrm{HD}$ (Lazic et al., 2004; Gil et al., 2005; Kohl et al., 2007; Kandasamy et al., 2010). Impaired neurogenesis was also found in several other neurodegenerative disorder models, such as models of Parkinson's disease and Alzheimer's disease (Haughey et al., 2002; Hoglinger et al., 2004; Wen et al., 2004; Donovan et al., 2006; Winner et al., 2008). In addition to modulating cell proliferation, pathological conditions might severely compromise the survival capacity of newly generated neurons. Recent studies have indicated a decrease of the long-term survival chance of newborn neurons by more than 80\% (Winner et al., 2004, 2008; Kohl et al., 2007; Marxreiter et al., 2009; Kandasamy et al., 2010). These observations underscore the incapacity of the endogenous mechanisms to achieve 
brain repair after injuries of the central nervous system. Our understanding of neurogenesis and its potential for functional brain repair still remain rudimentary.

A significant fraction of our knowledge of adult neurogenesis has been gained so far from static analyses. For example, the most prevailing technique to assess neurogenesis consists in injecting the thymidine analog 5-bromo-2'-deoxyuridine (BrdU), which integrate in newly synthesized DNA of proliferating cells. As further immunohistological analyses are required for the detection and phenotyping of cells labeled with BrdU, neurogenesis measurements are restricted to a single time point per individual. In order to understand the functional relevance of neurogenesis modulation and its potential role in the etiology of neurological and psychiatric disorders, it is required to assess neurogenesis repeatedly in a fashion as minimally invasive as possible. Consequently, over the past years sustained effort has been dedicated to develop imaging techniques to monitor adult neurogenesis in vivo.

From a clinical perspective, it would be advantageous to develop imaging procedures based on the use of currently available equipment, i.e., magnetic resonance imaging (MRI) and positron emission tomography (PET), as it could be more readily implanted in the clinics. Yet, for preclinical research, the exorbitant costs associated with these imaging devices limit their accessibility. The development of optical imaging procedures and devices partially filled the needs for lowcost imaging alternatives. This review summarizes the recent progress made in the field of in vivo imaging of neurogenesis and points to the strengths and weaknesses of the various strategies (Table 1).

\section{IN VIVO IMAGING OF NEUROGENESIS WITH MAGNETIC RESONANCE IMAGING AND SPECTROSCOPY}

The main advantage of MRI is its high spatial resolution that results in clear anatomical information. MRI uses a high magnetic field to align the nuclear magnetization of hydrogen atoms, or protons, of water in the body. Submitting a radio frequency pulse at the resonance frequency of these protons will cause the net magnetic vector to turn over into the plane perpendicular with the magnetic field.
The oscillation of this net magnetic vector about the main magnetic field will induce an electromotive force in the radio frequent (RF) antenna, i.e., the MR signal. Depending on the applied MRI pulse sequence, a specific MRI parameter will dominate the contrast in the images (i.e., contrast weighting). For most clinical examinations both $\mathrm{T}_{1}$-weighted and $\mathrm{T}_{2}$-weighted images are acquired for observation of white and gray matter brain structures. Other types of MRI contrast correlate with the diffusion of water molecules (i.e., Brownian motion) in biological tissues (Le Bihan et al., 1986) and are quite valuable in studying neuropathology related changes in intra/extracellular water balance and subcellular structural changes (Gass et al., 2001).

Magnetic resonance imaging contrast can be improved by applying contrast agents based on relaxivity (e.g., gadolinium chelates) or magnetic susceptibility (e.g., iron oxide particles, iron containing proteins). When placed in a magnetic field, iron oxide particles have been shown to induce local field inhomogeneities. These inhomogeneities shorten the $\mathrm{T}_{2}$ relaxation time of protons within a large perimeter, which results in hypointense (dark) signals in conventional $\mathrm{T}_{2} / \mathrm{T}_{2}{ }^{*}$-weighted imaging and therefore iron oxide particles are referred to as MRI-negative contrast agents (Norman et al., 1992; Bulte et al., 1999).

\section{DIRECT LABELING USING IRON OXIDE PARTICLES}

In order to enable cellular MRI, cells of interest have to be labeled with MR contrast agents. One of the options is the use of exogenous contrast agents. Numerous reports have demonstrated that iron oxide particles (from nanometer to micrometer size range) could be readily engulfed in vitro by cells of various origins, including neural stem and progenitor cells (for excellent reviews see Bulte et al., 2002; Bulte and Kraitchman, 2004; Frank et al., 2004; Modo et al., 2005). This approach constitutes an efficient strategy to label cells for detection as negative contrast. Results from agar phantoms containing iron oxide particle-labeled cells at various densities suggested that detection down to a single cell might be possible using high-resolution MRI (Kustermann et al., 2008). Previous studies

Table 1 | Comparison of various imaging methods for in vivo monitoring of adult neurogenesis.

\begin{tabular}{|c|c|c|c|c|c|c|}
\hline Technique & Optical Imaging & PET & MRI & & & \\
\hline Resolution & Low & Low & High & & & \\
\hline Sensitivity & $\begin{array}{l}\text { Limited depth } \\
\text { penetration }\end{array}$ & High & High & & & \\
\hline Human applicability & No & Yes & Yes & & & \\
\hline Method & $\begin{array}{l}\text { Fluorescence- } \\
\text { based imaging }\end{array}$ & $\begin{array}{l}\text { Bioluminescence- } \\
\text { based imaging }\end{array}$ & $\begin{array}{l}\text { Isotope-labeled } \\
\text { molecules }\end{array}$ & Iron oxide particles & MR reporter-genes & $\begin{array}{l}\text { MR } \\
\text { spectroscopy }\end{array}$ \\
\hline Technique & Genetic labeling & Genetic labeling & Direct labeling & Direct labeling & Genetic labeling & / \\
\hline $\begin{array}{l}\text { Neurogenesis- } \\
\text { specificity }\end{array}$ & $\begin{array}{l}\text { High with use of } \\
\text { specific promoters }\end{array}$ & $\begin{array}{l}\text { High with use of } \\
\text { specific promoters }\end{array}$ & Low & Low & $\begin{array}{l}\text { High with use of } \\
\text { specific promoters }\end{array}$ & Low \\
\hline Toxicity & No & No & $?$ & Yes & $?$ & No \\
\hline Cell-viability & Yes & Yes & Yes & No & Yes & Yes \\
\hline Limitations & $\begin{array}{l}\text { Signal scatter, } \\
\text { background } \\
\text { fluorescence }\end{array}$ & Signal scatter & $\begin{array}{l}\text { Exposure to } \\
\text { radiation }\end{array}$ & $\begin{array}{l}\text { Inhomogeneity artifact, } \\
\text { contrast dilution upon } \\
\text { cell division }\end{array}$ & $\begin{array}{l}\text { Low detection- } \\
\text { sensitivity }\end{array}$ & $\begin{array}{l}\text { Partial volume } \\
\text { artifact }\end{array}$ \\
\hline
\end{tabular}

$?=$ remains to be determined; / = no labeling required . 
have also shown that MRI is a useful tool to follow labeled, transplanted cell migration in the brain (Hoehn et al., 2002; Jendelova et al., 2003; Ben-Hur et al., 2007; Cohen et al., 2010).

Based on the observations that neural stem and progenitor cells could be loaded with iron oxide particles in vitro, various groups intended to label these cell populations by injecting iron oxide particles directly into the lateral ventricles (Shapiro et al., 2006a; Panizzo et al., 2009; Sumner et al., 2009; Vreys et al., 2010) or in the subventricular regions (Nieman et al., 2010; Vreys et al., 2010). This in situ labeling strategy proved to be successful, as a fraction of the injected particles were taken up by neuroblasts and carried away toward the OB (Figure 1). Hence permitting to follow neuronal migration along the RMS and into the $\mathrm{OB}$ as a read-out for neurogenesis and/or cell survival and migration.

Despite the valuable information on cell localization and migration dynamics, studies based on iron oxide labeled cells have several intrinsic limitations, with the most relevant one being the fact that iron oxide particles are passive contrast agents. The detected contrast on MR images refers only to the particle, and does not give any information on the cell-type or viability of the labeled cells, which leads to the possibility of non-specific findings (Slotkin et al., 2007; Schafer, 2010). Considering that more than half of the newly generated neurons die as they fail to integrate in their target regions (Winner et al., 2002; Kempermann et al., 2003), the extensive release of confounding particles arising from this cellular pruning would be far from incidental. Following death of the former carrier-cells, intracellular contrast particles will simply get deposited extracellular. These particles could be taken up by surrounding cells, such as microglia, and be carried away in an absolutely neurogenesis-independent fashion. Moreover, extracellular particles can be transported on cell surfaces and thereby migrate away from the injection site. Vreys et al. (2011) investigated the ability of magnetoliposomes (MLs) to label endogenous NPCs after direct injection into the adult mouse brain. Whereas MRI revealed contrast relocation toward the OB, the relocation was found to be independent of the migration of endogenous NPCs and represented background migration of MLs along white matter tracts. It was suggested that the small size of the MLs intrinsically limits their potential for in situ labeling of NPCs (Vreys et al., 2011).

Another limitation of particle-based cell labeling is the progressive dilution of the contrast medium with cell division. Cellular uptake of iron oxide particles occurs through endocytosis, which results in the accumulation of particles in the cytoplasm. As a result, the concentration of iron oxide particles halves with every cell division, leading to a progressive decrease in sensitivity to detect labeled cells (Magnitsky et al., 2005). While the use of micron-sized iron oxide particles (MPIOs) allows visualization of cells containing a single particle (Shapiro et al., 2006b), cell progeny after further proliferation of the cells will not be labeled with contrast particles. This dilution effect upon cell division is a major drawback for the longitudinal follow-up of highly proliferating cells.

In case of direct in situ labeling, the labeling efficiency is quite low and only a small fraction of targeted cells will be labeled with the contrast agent (Sumner et al., 2009; Nieman et al., 2010). In addition, the engulfment of iron oxide particles is rather unspecific. For example, immunohistochemistry and electron microscopy showed that after direct injection of MPIOs in the lateral ventricle, contrast particles were not only located inside the targeted celltype, i.e., neural stem and progenitor cells, but also in ependymal cells, microglia, and oligodendrocyte progenitor cells (Shapiro et al., 2006a; Sumner et al., 2009). Careful analysis of the nature of labeled cells suggests that the site of injection is defining the subpopulation of cells labeled with the contrast particles (Nieman et al., 2010). The fact that the phenotype of cells internalizing the particles can only be determined a posteriori by histological analysis, is particularly troublesome for longitudinal studies, taken that labeled neural stem and progenitor cells are multipotent and may undergo extensive differentiation processes comprising multiple transient maturation stages.

Currently, the use of in situ labeling of endogenous neural stem and progenitor cells is restricted to the neurogenic niche of the SVZ. The reason for this restriction is that the amount of iron oxide particles required for in situ labeling generates substantial

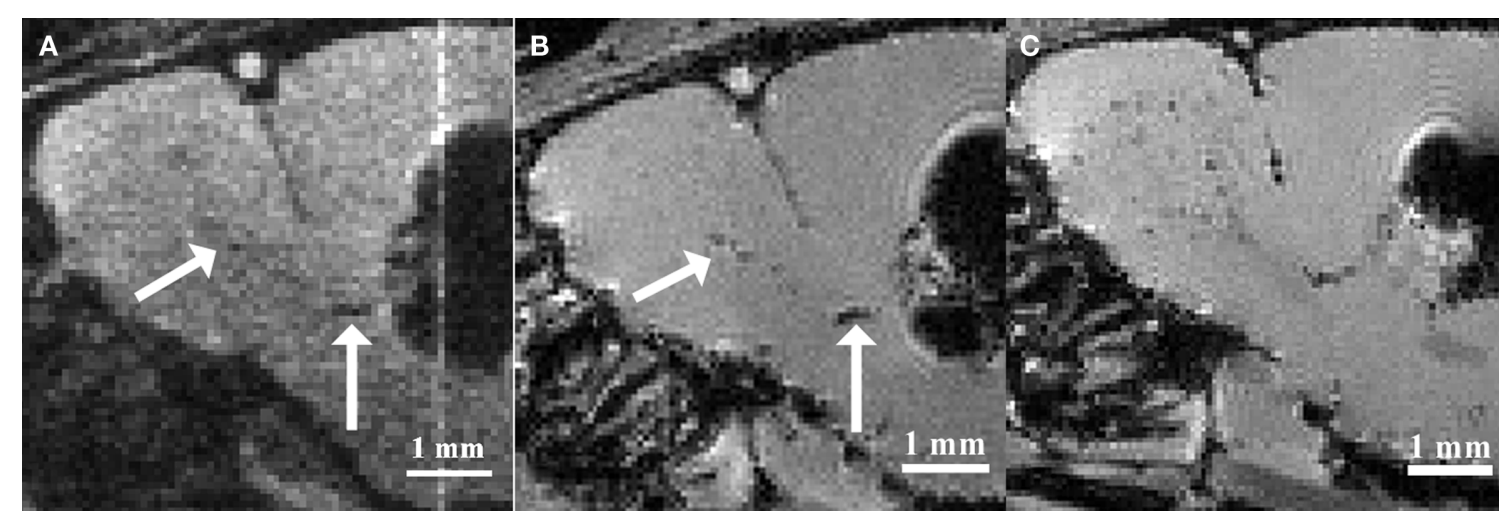

FIGURE 1 | Magnetic resonance imaging of in situ labeled neural precursor cell migration. (A) In vivo MRI of a mouse injected with micron-sized iron oxide particles in the lateral ventricle at 3 weeks post-injection. (B) Ex vivo MRI of the same mouse at 3 weeks post-injection. (C) Ex vivo MRI of a mouse injected with micron-sized iron oxide particles in the lateral ventricle at 8 weeks post-injection. Arrows indicate corresponding hypointense contrast on the in vivo and ex vivo MRI of the same mouse (Adapted and reproduced from Vreys et al., 2010). 
image distortion, i.e., susceptibility inhomogeneity, at the site of injection (Shapiro et al., 2006a; Vreys et al., 2010). As neuroblasts from the SGZ migrate into the directly overlying granule cell layer, the susceptibility inhomogeneity would hamper the visualization of the labeled cell migration.

Cell labeling using non-degradable iron oxide particles, such as MPIOs, is permanent, and careful evaluation of long-term toxicity caused by labeling compounds becomes critical. Although limited toxicity has been demonstrated following in vitro labeling (Crabbe et al., 2010), studies on in vivo application of iron oxide particles have recently revealed decreased cell proliferation and migration, as well as signs of inflammation (Schafer, 2010; Vreys et al., 2010). Unfortunately, in most reports the assessments of the impact of iron oxide particles on gene expression and cell fate have remained cursory.

The majority of studies on MR imaging of endogenous neural stem and progenitor cells are performed so far in naive and healthy animals. A recent study showed that in situ labeling of endogenous neural stem and progenitors cells with MPIOs could reveal altered cell migration toward an hypoxic-ischemic insult using in vivo MRI (Yang et al., 2009). The proof-of-principle should now be extended to various disease models. Undoubtedly, the feasibility of iron oxide particle labeling for long-term imaging of neurogenesis in clinically relevant applications should be fully investigated. It is expected that the modified cell composition of the neurogenic niche (e.g., presence of reactive gliosis, macrophage infiltrations, etc.) as observed in pathological conditions as well as the possibility of reduced survival of new neurons induced by the pathological conditions, as mentioned earlier, would complicate neurogenesisspecific labeling and its intended imaging.

\section{GENETIC LABELING USING MRI REPORTER-GENES}

In order to overcome the disadvantages of iron oxide particle labeling, a new approach using MRI reporter-genes has recently been developed. Genetic reporters can be incorporated in gene delivery systems like viral vectors or in transgenic animals. This labeling approach solves the problem of contrast agent dilution and subsequent signal loss upon cell division. In addition, vector-mediated reporter-gene delivery holds the potential for specific labeling of neuroblasts by the use of specific promoters. Another advantage is that the transgene construct can be coupled with an additional transgene, for example a therapeutic one, or with other reportergenes for multimodality imaging.

A promising reporter for MRI is ferritin, a ubiquitously expressed metalloprotein, assembled out of 24 light and heavy subunits (Cohen et al., 2005; Genove et al., 2005). Ferritin sequesters endogenous iron from the organism and stores it in a hydrated iron oxide (ferrihydrite) core that significantly affects the $\mathrm{T}_{2}$ relaxation times of protons (Gossuin et al., 2004). The corresponding changes in contrast on $\mathrm{T}_{2}$ - and $\mathrm{T}_{2}{ }^{*}$-weighted $\mathrm{MR}$ images could be a quantitative read-out for both neuronal proliferation and recruitment to the target region. Furthermore, the absence of image distortion (such as seen at the iron oxide particle injection area) would allow targeting the dentate gyrus of the hippocampus as well as the SVZ. To date, however, the low detection-sensitivity limits the use of current transgenic systems in cell tracking applications (Vande Velde et al., 2011). It was also reported that the viral vector systems used as delivery systems themselves can elicit ferritin-like hypointense contrast on MR images (Vande Velde et al., 2011). Enhancement of the sensitivity of MR reporter systems will remain a challenge and future studies should focus not only on optimization of the vector constructs for the delivery of MR reporter-genes, but also on improving the sensitivity to detect reporter-gene based contrast both at the acquisition and at the post-processing level.

Another MRI reporter candidate is magA, a gene known to be involved with iron transport in magnetotactic bacteria. Zurkiya et al. (2008) and Goldhawk et al. (2009) demonstrated that magA transfected mammalian cells formed in vivo magnetic iron oxide nanoparticles which allowed subsequent visualization of the cells with MRI (Zurkiya et al., 2008; Goldhawk et al., 2009). Whether magA is an appropriate MRI reporter-gene for endogenous stem cell labeling needs to be further investigated.

Although these approaches are attractive in that they combine the cell specificity of the transgenic system with the high-resolution of MR imaging, possible toxicity associated with the accumulation of iron in the cytoplasm must be carefully evaluated. Evidence for toxicity has been provided by recent reports demonstrating that in a transgenic model, long-term expression of elevated ferritin levels in neurons may lead to age-associated neurodegeneration (Kaur et al., 2007, 2009).

\section{MAGNETIC RESONANCE SPECTROSCOPY}

Magnetic resonance spectroscopy (MRS) is an MR technique that can measure brain biochemistry. In this way, it adds nicely to other MRI applications as it provides metabolic information in addition to anatomical, physiological, functional, or molecular imaging information. Localized ${ }^{1} \mathrm{H}$-MRS currently allows for the quantification of more than $18{ }^{1} \mathrm{H}$ containing metabolites in vivo (Pfeuffer et al., 1999). The complexity of an MR spectrum can be appreciated in Figure 2 in which a region of an MR spectrum

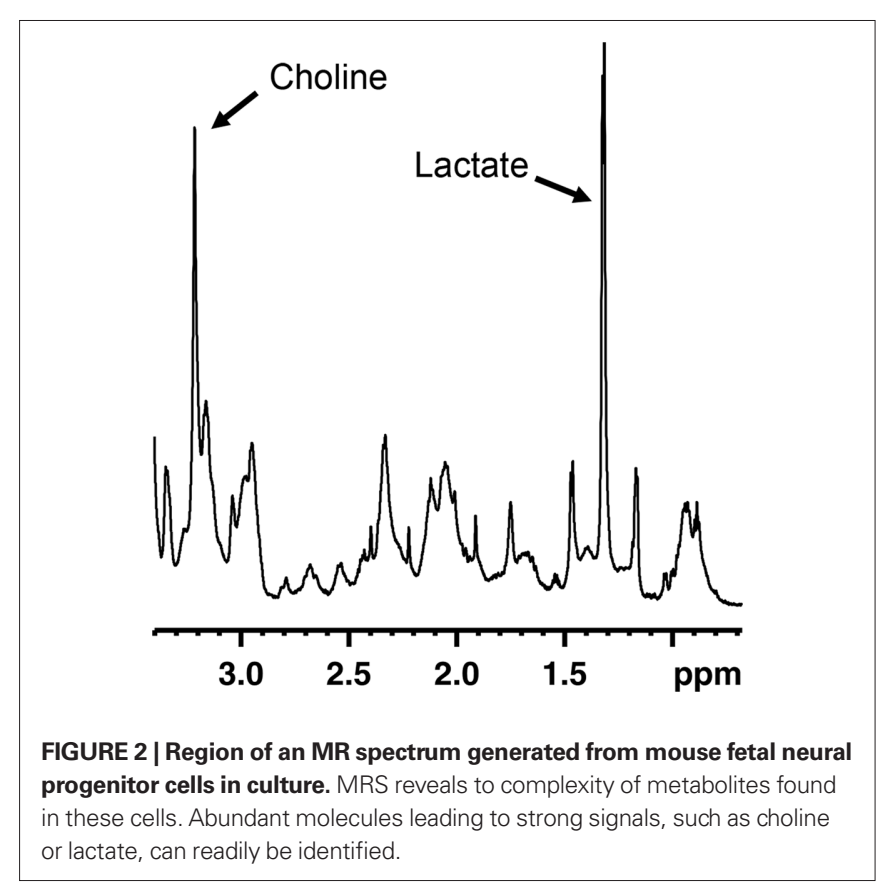


measured on mouse fetal neural stem and progenitor cells is represented. Localized MRS techniques allow assessment of metabolites comprised in a well-defined volume/voxel within the brain and can return single-volume, or multi-volume information with microliter resolution. This constitutes an attractive strategy to appreciate the rate of neurogenesis in a non-invasive fashion. If there would be a molecule specific or enriched in the neurogenic niche, it would be theoretically possible to detect a spectroscopic signature proper to this molecule in voxels located within neurogenic regions. The intensity of the neurogenesis-associated peak would reveal the "concentration of neural stem or progenitor cells" within the voxel.

Manganas et al. (2007) reported the identification of an MRS peak, detected on the spectrum at $1.28 \mathrm{ppm}$, which seemed to correlate with the presence of neural stem/progenitor cells in the dentate gyrus of both humans and rats. In vitro, this peak was particularly abundant in undifferentiated neural stem cells, and appeared to be absent or weak in neurons, astrocytes, and oligodendrocytes. Interestingly, it was possible to detect and localize in vivo implanted neural stem cells in the cortex of rats, based on the 1.28-ppm peak. In addition, Manganas et al. (2007) demonstrated that in agreement with the expected age-related decrease of neurogenesis the intensity of the 1.28-ppm peak measured within the hippocampus was undergoing a dramatic decrease when comparing recordings performed in 8 to 10-year-old children with those of 30 to 35-year-old adults. Although the report from Manganas et al. (2007) promptly put MRS back into the limelight, several researchers question the specificity of the 1.28 -ppm, as well as the capacity to detect the stem and progenitor cells using the equipment currently available (Friedman, 2008; Hoch et al., 2008; Jansen et al., 2008; Dong et al., 2009; Ramm et al., 2009). A recent study, which aimed to identify the origin of the 1.28-ppm peak, demonstrated that this spectroscopic signal correlates with the presence of mobile lipid droplets within cells (Ramm et al., 2009), a phenomenon observed during cell proliferation and apoptosis regardless of the cell-type (e.g., neural stem cells, COS7 cells, etc.). This may turn the 1.28-ppm signal into a correlated peak of the neurogenic niches rather than as a specific marker for neurogenesis per se.

The greatest advantage of MRS is the complete non-invasive nature of the protocol. For measurements in the SVZ, however, its close lining to the ventricles filled with cerebrospinal fluid constitutes an extra challenge. When an MRS voxel contains a mixture of tissue types the spectroscopic signal will also consist of signals from different tissues (i.e., a partial volume artifact). Since large voxel sizes are currently required to acquire sufficient signal-to-noise ratios, performing a reliable measurement of the SVZ is a difficult task. Further technical developments are warranted to reduce the voxel size targeted during spectroscopic analysis and to increase the sensitivity with the main objective to focus exclusively on neurogenic regions and thereby to increase the "stem cell concentration" and detectability.

\section{IN VIVO IMAGING OF NEUROGENESIS WITH POSITRON EMISSION TOMOGRAPHY}

Positron emission tomography might not have the resolution of MRI, however, it shows an extraordinary sensitivity, which is a powerful advantage considering the relatively scarceness of stem cells in the adult brain. PET relies on the administration of radioactive isotope-labeled molecules designed to bind to or concentrate themselves at the specified target sites. Positron-emitting radionuclides, such as $\left[{ }^{18} \mathrm{~F}\right]$ or $\left[{ }^{11} \mathrm{C}\right]$, are selected for labeling since upon annihilation of a positron with an electron from the body, two gamma photons with an energy of $511 \mathrm{keV}$ are emitted in diametrically opposite directions. Registration of these so-called coincident photons, i.e., pairs of photons reaching quasi simultaneously the sensitive photodetectors ringing the subject, allows for the computation of position and intensity of the emission source(s).

Rueger et al. (2010) recently reported the in vivo detection of cell proliferation within the rat neurogenic niches based on $3^{\prime}$-deoxy- $3^{\prime}$ $\left[{ }^{18} \mathrm{~F}\right]$ fluoro-L-thymidine $\left(\left[{ }^{18} \mathrm{~F}\right]\right.$-FLT $)$ signals (Figure 3$) .\left[{ }^{18} \mathrm{~F}\right]$-FLT is a thymidine analog and can be regarded as a PET equivalent to BrdU used in histological analysis. Even though Rueger et al. (2010) also reported that modulated proliferation rates following pharmacological or surgical interventions could be detected, $\left[{ }^{18} \mathrm{~F}\right]$-FLT signal intensities emanating from the SVZ and SGZ appeared similar to each other although it is known that the magnitude of proliferation in the SVZ is from one to two orders higher than in the SGZ. Hence, these findings suggest that binding of $\left[{ }^{18} \mathrm{~F}\right]$-FLT is determined by additional parameters than solely neural stem and progenitor cell proliferation. Thus, great care should be taken when comparing signals from different sources.

Furthermore, some other issues remain to be resolved before PET can be reliably used to monitor neurogenesis in vivo. For example, the identity of cells at the origin of the $\left[{ }^{18} \mathrm{~F}\right]$-FLT signals cannot be inferred by this technique. This precludes the use of $\left[{ }^{18} \mathrm{~F}\right]$-FLT for neurogenesis imaging under pathological conditions, since proliferation associated with reactive gliosis or immune cell infiltrations would be indistinguishable from neural stem/progenitor cell proliferation. Nonetheless, the work of Rueger et al. (2010) constitutes a valuable proof-of-principle that PET could be used for in vivo imaging of neurogenesis in the future. The development of neurogenesis-specific labeling compounds currently constitutes an important limiting step for this achievement.

\section{IN VIVO IMAGING OF NEUROGENESIS WITH OPTICAL IMAGING}

Over the last decade, optical imaging techniques became widespread in numerous preclinical research fields, including the study of neurogenesis. The possibility to combine several labels simultaneously, as well as the broad availability of current transgenic technologies, made optical imaging a very attractive method. Optical imaging can be based on the detection of fluorescent or bioluminescent signals, both bearing their strengths and limitations.

\section{FLUORESCENCE-BASED IMAGING OF NEUROGENESIS}

The simplicity of fluorescence-based imaging and the minimal requirement in equipment explain the extensive usage of this technique. The method is based on the capacity of some fluorescent molecules to reflect specific incident wavelengths with a red shift. The incident and reflected light can be separated using an appropriate sets of passfilters. Components for fluorescence imaging are frequently coupled with microscopes, resulting in an imaging resolution at the level of the cell and even subcellular elements (Figure 4). This high-resolution is an advantage for the study of neurogenesis, since not only the position and amount of cells, but 

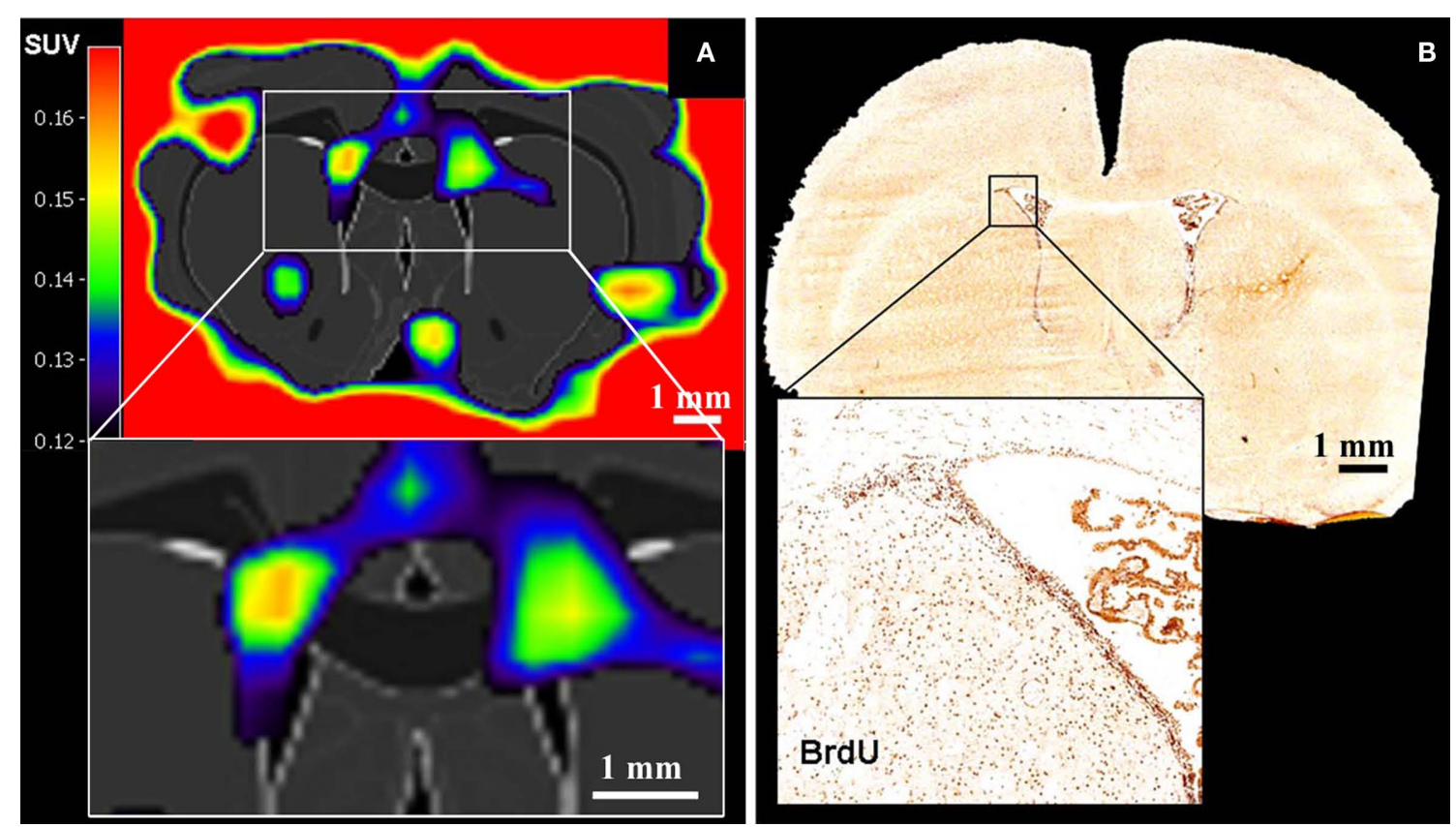

FIGURE 3 | Positron emission tomography imaging of neural stem cell proliferation ([18F]-FLT-PET matched on MRI-atlas of rat brain). (A) Increased [ $\left.{ }^{18} \mathrm{~F}\right]-\mathrm{FLT}$ signal was detected in the SVZ. (B) Location of BrdU-labeled proliferating cells in the SVZ corresponds well with the elevated [18F]-FLT signal in the SVZ (A) (Adapted from Rueger et al., 2010)
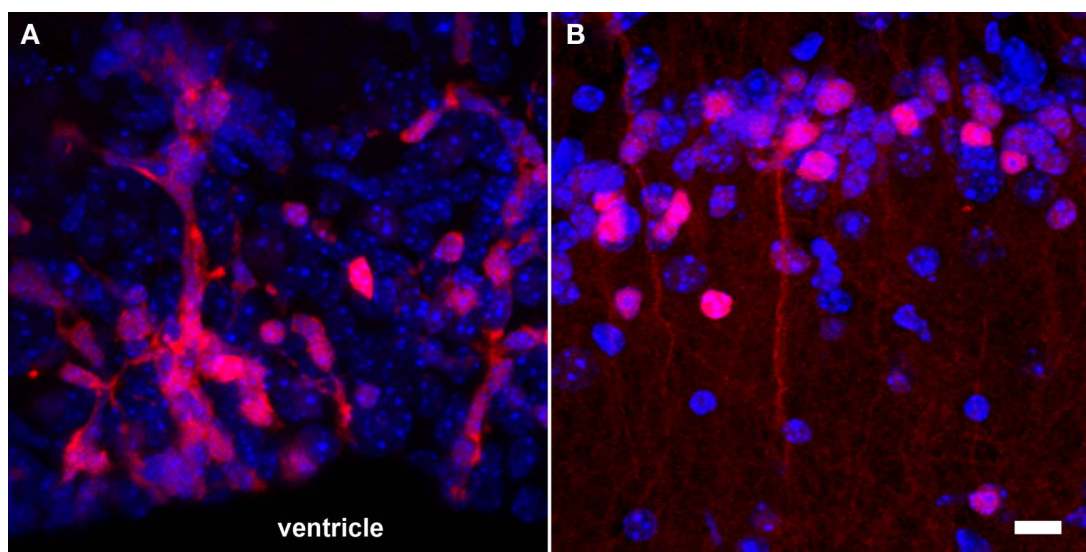

FIGURE 4 | Fluorescence-based imaging of neurogenesis using transgenic expression of DsRed under the control of the doublecortin promoter. (A) A large number of neuronal precursors (red) are generated in the SVZ and migrate in toward the olfactory bulb along the RMS; (B) once neuroblasts (red) reach the granular cell layer of the olfactory bulb, they start to functionally integrate and have a more complex cellular morphology. Nuclei appear in blue. Scale bar $=10 \mu \mathrm{m}$.

also the morphology and the integration of newly generated neurons are highly relevant. However, high-resolution fluorescence imaging can only be performed on relatively thin samples. As a result, neurogenesis is generally analyzed on acute brain slices, which can be maintained alive in vitro for only a few hours. In case of whole body in vivo imaging, where animals are placed in a dark chamber equipped with an illumination source and a sensitive photodetector system, the spatial resolution is rather low because the light reflected from deep structures is scattered before it reaches the body surface.
The use of fluorescent protein expression, such as the green fluorescent protein (GFP), was a breakthrough for in vivo imaging of biological processes (Heim et al., 1994). Fluorescent reporters have the advantage that once encoding transgenes have been introduced within the genome, fluorescent signals are generated throughout life without further need for additional labeling. Transgenic mouse and rat models for the analysis of neurogenesis have been generated over the last years using cell-type-specific promoters to control the expression of various fluorescent proteins. For example, transgenic models have been developed using the nestin and the doublecortin 
promoters to detect neural stem and progenitor cells respectively (Yamaguchi et al., 2000; Karl et al., 2005; Couillard-Despres et al., 2006, 2008b; Ladewig et al., 2008). Besides the transgenic models, the delivery of retroviral vectors encoding fluorescent proteins to neurogenic regions has also been fruitfully exploited (Carleton et al., 2003; van Praag et al., 2005; Toni et al., 2008). Taking advantage of the proliferation capacity of neural stem and progenitor cells, it is possible to label a sub-population of these cells permanently and rather selectively using retroviral vectors. Moreover, using the fluorescent signal to target recording electrodes, synaptic integration of newly generated neurons can be investigated by electrophysiology (Carleton et al., 2003; Couillard-Despres et al., 2006; Toni et al., 2008).

One general concern for in vivo fluorescent imaging is the autofluorescence of biological tissues. In addition, the shallow tissue penetration at which high quality imaging is currently achievable by in vivo fluorescence imaging constitutes a major hindrance to the analysis of neurogenesis. Even in relatively small animals such as mice, the hippocampus and subventricular regions are too deep within the brain for conventional microscopy. Significant improvement has already been obtained using multiphoton confocal microscopy, which expands the observation window to a depth of approximately $500 \mu \mathrm{m}$ (Fuhrmann et al., 2007), yet this is barely enough for the analysis of the mouse cerebral cortex. Furthermore, signal scattering will always be a limiting factor for accurate recording, especially when light is reflected from regions lying deep in tissues. Thus the cellular resolution of fluorescence imaging obtained in vitro using conventional microscopy is beyond the limits of current whole body in vivo imaging. Optical tomography, might significantly improve the quality and precision of fluorescence imaging, in a fashion similar to PET acquisitions (Garofalakis et al., 2007). To improve the detectability of fluorescent reporters, bright redshifted fluorescent proteins, such as tdTomato, mCherry, mPlum, or Katushka have been developed over the last years to take advantage of a good light transmission window in the far-red region of the visible light spectrum, from approximately 600 to $850 \mathrm{~nm}$ (Shaner et al., 2004; Deliolanis et al., 2008).

\section{BIOLUMINESCENCE-BASED IMAGING OF NEUROGENESIS}

The use of various luciferase reporter-genes for bioluminescent optical imaging is another technique that has recently gained interest. Enzymes of the luciferase family are not evolutionary related, but they all cause photons to be emitted upon catalysis of their respective substrates. These photons can be acquired and processed for imaging purposes. The luciferase isolated from the firefly (Photinus pyralis) and the sea pansy (Renilla reniformis) are the most widely distributed among the various luciferases identified and cloned so far. However, the substrate catalyzed by the Renilla luciferase, i.e., coelenterazine, presents an imidazopyrazine structure that can promptly auto-oxidize. This auto-degradation process is further enhanced by the presence of albumin (Zhao et al., 2004). Thus, the structural instability of coelenterazine could lead to elevated background signals during in vivo imaging of weak reporter activities. In addition, coelenterazine can be re-transported out of cells by the multidrug resistance MDR1 P-glycoprotein (Pgp), which could generate unforeseen variability in signal intensities between various cell populations exposed to the same extracellular coelenterazine concentration (Pichler et al., 2004). Therefore, the firefly's luciferase is currently the most broadly used enzyme for in vivo bioluminescence imaging in mice and rats.

During in vivo bioluminescence imaging, animals are placed in a dark chamber equipped with an extremely sensitive photodetector system. In contrast to fluorescent imaging, the appropriate substrate (e.g., D-luciferin in the case of the firefly luciferase) needs to be injected shortly before recording in order to achieve a detectable bioluminescent signal. Since each organ or body compartment is supplied differently, the regional distribution, catalysis, and elimination kinetics of the substrate must be taken into account in the design and execution of the work (Lee et al., 2003).

The use of bioluminescence for imaging of neurogenesis resolves some problems discussed previously with the use of iron oxide particles in MRI. For example, bioluminescence recording guaranties viability of cells at the origin of the reporter signal since the generation of photons resulting from the catalysis of D-luciferin is ATP-dependent. In addition, there have been so far no reports on toxicity or inflammation elicited by the firefly's luciferase or its substrate d-luciferin. As a result, the impact on neurogenesis is assumed to be minimal. Nonetheless, this topic requires further investigation in order to fully verify this assumption.

Although the spatial resolution of bioluminescence imaging is similar to that of fluorescence imaging due to signal scatter from deep brain regions, it has one solid advantage over fluorescence-based imaging. The virtual absence of bioluminescence background from tissues allows for a greater sensitivity of detection, as required for neural stem and progenitor cells. Both grafting of transgenic neural stem cells and in situ retroviral labeling of endogenous neural stem cells in wild type mice have been shown to result in a very high signal/noise ratio (Couillard-Despres et al., 2008a; Reumers et al., 2008). The fate of a small group of transgenic neural stem cells implanted in the subventricular region of a wild type mouse could be followed over several days during their journey toward the $\mathrm{OB}$ (Figure 5). Moreover, the use of a neuronal precursor-specific promoter to drive luciferase expression, such as a doublecortin promoter, guarantees that the bioluminescence is neurogenesis-specific (Couillard-Despres et al., 2008a).

Even though the use of specific promoters can selectively target specific cell populations, such transgenic systems remain confined to preclinical research. Furthermore, given the large size of the

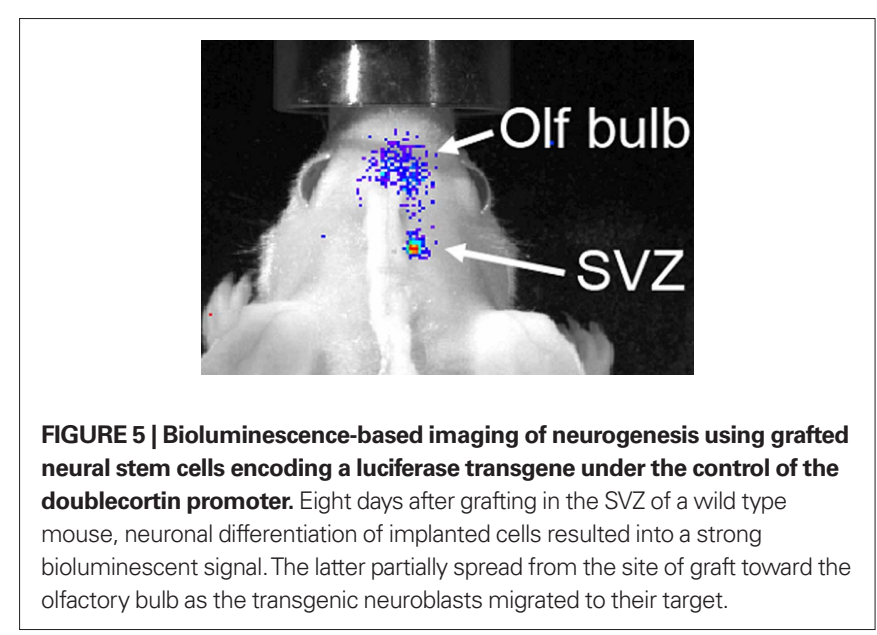


human brain, it is unlikely that optical techniques will be suited for in vivo imaging of human neurogenesis. Nevertheless, optical imaging remains a powerful and valuable method in preclinical research for the in vivo imaging of neurogenesis.

\section{CONCLUSION}

Over the last decade, tremendous progress has been made in the development of strategies for in vivo imaging of neurogenesis. With the exception of MRS, all imaging modalities discussed in this review rely on either direct labeling (e.g., iron oxide particles, $\left[{ }^{18} \mathrm{~F}\right]$-FLT) or indirect labeling (i.e., the use of reporter-genes) to achieve visualization of cells contributing to neurogenesis. Although both labeling strategies have shown their potentials for in vivo imaging, the major challenges to achieve are neurogenesis-specificity and detection-sensitivity for direct and indirect labeling respectively. Beside these challenges one must always carefully evaluate safety requirements. Therefore, the toxicity and interference associated with labels applied or genes expressed should be addressed with great care. Moreover, in cases of direct labeling, labels must not only be non-toxic, but in view of future clinical applications, should preferentially be biodegradable as well.

Although MRS does not encounter the problems related with cell labeling, proof of specific visualization of neurogenesis by this modality has still to be provided. Briefly, none of the imaging strategies has so far been able to fulfill all needs and expectations; they all have their advantages and disadvantages

\section{REFERENCES}

Altman, J., and Das, G. D. (1965). Autoradiographic and histological evidence of postnatal hippocampal neurogenesis in rats. J. Comp. Neurol. 124, 319-335.

Arvidsson, A., Collin, T., Kirik, D., Kokaia, Z., and Lindvall, O. (2002). Neuronal replacement from endogenous precursors in the adult brain after stroke. Nat. Med. 8, 963-970.

Ben-Hur, T., van Heeswijk, R. B., Einstein, O., Aharonowiz, M., Xue, R., Frost, E. E., Mori, S., Reubinoff, B. E., and Bulte, J. W. M. (2007). Serial in vivo MR tracking of magnetically labeled neural spheres transplanted in chronic EAE mice. Magn. Reson. Med. 57, 164-171.

Bulte, J. W., Brooks, R. A., Moskowitz, B. M., Bryant, L. H. Jr., and Frank, J. A. (1999). Relaxometry and magnetometry of the MR contrast agent MION46L. Magn. Reson. Med. 42, 379-384.

Bulte, J. W., Duncan, I. D., and Frank, J. A. (2002). In vivo magnetic resonance tracking of magnetically labeled cells after transplantation. J. Cereb. Blood Flow Metab. 22, 899-907.

Bulte, J.W., and Kraitchman, D. L. (2004). Monitoring cell therapy using iron oxide MR contrast agents. Curr. Pharm. Biotechnol. 5, 567-584.

Cameron, H. A., Woolley, C. S., McEwen, B. S., and Gould, E. (1993).
Differentiation of newly born neurons and glia in the dentate gyrus of the adult rat. Neuroscience 56, 337-344.

Carleton, A., Petreanu, L. T., Lansford, R., Alvarez-Buylla, A., and Lledo, P. M. (2003). Becoming a new neuron in the adult olfactory bulb. Nat. Neurosci. 6 , 507-518.

Cohen, B., Dafni, H., Meir, G., Harmelin, A., and Neeman, M. (2005). Ferritin as an endogenous MRI reporter for noninvasive imaging of gene expression in C6 glioma tumors. Neoplasia 7, 109-117.

Cohen, M. E., Muja, N., Fainstein, N. Conserved fate and function of ferumoxides-labeled neural precursor cells in vitro and in vivo. J. Neurosci. Res. 88, 936-944.

Conover, J. C., and Allen, R. L. (2002). The subventricular zone: new molecular and cellular developments. Cell. Mol. Life Sci. 59, 2128-2135.

Couillard-Despres, S., Finkl, R., Winner, B., Ploetz, S., Wiedermann, D., Aigner, R., Bogdahn, U., Winkler, J., Hoehn, M., and Aigner, L. (2008a). In vivo optical imaging of neurogenesis: watching new neurons in the intact brain. Mol. Imaging 7, 28-34.

Couillard-Despres, S., Quehl, E., Altendorfer, K., Karl, C., Ploetz, S., Bogdahn, U., Winkler, J., and Bulte, J.W.M., and Ben-Hur, T. (2010).

regarding sensitivity, tissue penetration, etc. Nonetheless, the different approaches available are rather to be considered as complementary.

Similar to the use of BrdU or tritiated-thymidine in the earlier neurogenesis studies, assessment of cell proliferation does not constitute a valid measurement of neurogenesis. At this time, improvement of the detection using direct or indirect labeling of neuronal precursors and young neurons, which reflects the rates of neurogenesis, constitutes the next challenge (Couillard-Despres et al., 2005). Finally, although preclinical settings offer the possibility to use transgenic reporter systems, which are powerful tools for the understanding of neurogenesis under physiological and pathological contexts, the development of neurogenesis imaging techniques applicable to human remains the ultimate goal.

\section{ACKNOWLEDGMENTS}

The authors are grateful to Paul Ramm for fruitful discussion and for providing graphical material. The authors also wish to thank Dr. Greetje Vande Velde for her help with the manuscript. This work was supported in part by the State Government of Salzburg (Austria), the Bavarian State Ministry of Sciences, Research and Arts (ForNeuroCell2 grant), Inter University Attraction Poles (IUAP-NIMI-P6/38), the Flemish institute supporting scientific-technological research in industry (IWT-60838 BRAINSTIM), the University of Antwerp (Concerted Research Actions funding), and the European Commission for EC-FP6 network DiMI (LSHB-CT-2005-512146).

Aigner, L. (2008b). Human in vitro reporter model of neuronal development and early differentiation processes. BMC Neurosci. 9, 31. doi: 10.1186/1471-2202-9-31

Couillard-Despres, S., Winner, B., Karl, C. Lindemann, G., Schmid, P., Aigner, R., Laemke, J., Bogdahn, U., Winkler, J., Bischofberger, J., and Aigner, L. (2006). Targeted transgene expression in neuronal precursors: watching young neurons in the old brain. Eur. J. Neurosci. 24, 1535-1545.

Couillard-Despres, S., Winner, B. Schaubeck, S., Aigner, R., Vroemen, M., Weidner, N., Bogdahn, U., Winkler, J., Kuhn, H. G., and Aigner, L. (2005). Doublecortin expression levels in adult brain reflect neurogenesis. Eur. J. Neurosci. 21, 1-14.

Crabbe, A., Vandeputte, C., Dresselaers, T., Sacido, A. A., Verdugo, J. M. G., Eyckmans, J., Luyten, F. P., Van Laere, K., Verfaillie, C. M., and Himmelreich, U. (2010). Effects of MRI contrast agents on the stem cell phenotype. Cell Transplant. 19, 919-936.

Curtis, M. A., Penney, E. B., Pearson,A. G., van Roon-Mom, W. M., Butterworth, N. J., Dragunow, M., Connor, B., and Faull, R. L. (2003). Increased cell proliferation and neurogenesis in the adult human Huntington's disease brain. Proc. Natl. Acad. Sci. U.S.A 100, 9023-9027.
Curtis, M. A., Penney, E. B., Pearson, J., Dragunow, M., Connor, B., and Faull, R. L. (2005). The distribution of progenitor cells in the subependymal layer of the lateral ventricle in the normal and Huntington's disease human brain. Neuroscience 132, 777-788.

Deliolanis, N. C., Kasmieh, R., Wurdinger, T., Tannous, B. A., Shah, K., and Ntziachristos, V. (2008). Performance of the red-shifted fluorescent proteins in deep-tissue molecular imaging applications. J. Biomed. Opt. 13, 044008.

Dong, Z., Dreher, W., Leibfritz, D., and Peterson, B. S. (2009). Challenges of using MR spectroscopy to detect neural progenitor cells in vivo. AJNR Am. J. Neuroradiol. 30, 1096-1101.

Donovan, M. H., Yazdani, U., Norris, R. D., Games, D., German, D. C., and Eisch, A. J. (2006). Decreased adult hippocampal neurogenesis in the PDAPP mouse model of Alzheimer's disease. J. Comp. Neurol. 495, 70-83.

Eriksson, P. S., Perfilieva, E., BjorkEriksson, T., Alborn, A. M., Nordborg, C., Peterson, D. A., and Gage, F. H. (1998). Neurogenesis in the adult human hippocampus. Nat. Med. 4, 1313-1317.

Frank, J. A., Anderson, S. A., Kalsih, H. Jordan, E. K., Lewis, B. K., Yocum, G. T., and Arbab, A. S. (2004). Methods for magnetically labeling 
stem and other cells for detection by in vivo magnetic resonance imaging. Cytotherapy 6, 621-625.

Friedman, S. D. (2008). Comment on "magnetic resonance spectroscopy identifies neural progenitor cells in the live human brain." Science 321, 640.

Fuhrmann, M., Mitteregger, G., Kretzschmar, H., and Herms, J. (2007). Dendritic pathology in prion disease starts at the synaptic spine. J. Neurosci. 27, 6224-6233.

Garofalakis, A., Zacharakis, G., Meyer, H., Economou, E. N., Mamalaki, C., Papamatheakis, J., Kioussis, D., Ntziachristos, V., and Ripoll, J. (2007). Three-dimensional in vivo imaging of green fluorescent protein-expressing $\mathrm{T}$ cells in mice with noncontact fluorescence molecular tomography. $\mathrm{Mol}$. Imaging 6, 96-107.

Gass, A., Niendorf, T., and Hirsch, J. G. (2001). Acute and chronic changes of the apparent diffusion coefficient in neurological disorders-biophysical mechanisms and possible underlying histopathology. J. Neurol. Sci. 186(Suppl. 1), S15-S23.

Genove, G., DeMarco, U., Xu, H., Goins, W. F., and Ahrens, E. T. (2005). A new transgene reporter for in vivo magnetic resonance imaging. Nat. Med. 11, 450-454.

Gil, J. M., Mohapel, P., Araujo, I. M., Popovic, N., Li, J. Y., Brundin, P., and Petersen, A. (2005). Reduced hippocampal neurogenesis in R6/2 transgenic Huntington's disease mice. Neurobiol. Dis. 20, 744-751.

Goldhawk, D. E., Lemaire, C., McCreary, C. R., McGirr, R., Dhanvantari, S., Thompson, R. T., Figueredo, R., Koropatnick, J., Foster, P., and Prato, F. S. (2009). Magnetic resonance imaging of cells overexpressing MagA, an endogenous contrast agent for live cell imaging. Mol. Imaging 8, 129-139.

Gossuin, Y., Muller, R. N., and Gillis, P. (2004). Relaxation induced by ferritin: a better understanding for an improved MRI iron quantification. NMR Biomed. 17, 427-432.

Haughey, N. J., Nath, A., Chan, S. L., Borchard, A. C., Rao, M. S., and Mattson, M. P. (2002). Disruption of neurogenesis by amyloid betapeptide, and perturbed neural progenitor cell homeostasis, in models of Alzheimer's disease. J. Neurochem. 83, 1509-1524.

Heim, R., Prasher, D. C., and Tsien, R. Y. (1994). Wavelength mutations and posttranslational autoxidation of green fluorescent protein. Proc. Natl. Acad. Sci. U.S.A. 91, 12501-12504

Hoch, J. C., Maciejewski, M. W., and Gryk, M. R. (2008). Comment on "magnetic resonance spectroscopy identifies neural progenitor cells in the live human brain." Science 321, 640 .

Hoehn, M., Kustermann, E., Blunk, J., Wiedermann, D., Trapp, T., Wecker, S., Focking, M., Arnold, H., Hescheler, J., Fleischmann, B. K., Schwindt, W., and Buhrle, C. (2002). Monitoring of implanted stem cell migration in vivo: a highly resolved in vivo magnetic resonance imaging investigation of experimental stroke in rat. Proc. Natl. Acad. Sci. U.S.A. 99, 16267-16272.

Hoglinger, G. U., Rizk, P., Muriel, M. P., Duyckaerts, C., Oertel, W. H., Caille, I., and Hirsch, E. C. (2004). Dopamine depletion impairs precursor cell proliferation in Parkinson disease. Nat. Neurosci. 7, 726-735.

Jansen, J. F., Gearhart, J. D., and Bulte, J.W. (2008). Comment on "Magnetic resonance spectroscopy identifies neural progenitor cells in the live human brain." Science 321, 640.

Jendelova, P., Herynek, V., DeCroos, J. Glogarova, K., Andersson, B., Hajek, M., and Sykova, E. (2003). Imaging the fate of implanted bone marrow stromal cells labeled with superparamagnetic nanoparticles. Magn. Reson. Med. 50, 767-776.

Jin, K., Minami, M., Lan, J. Q., Mao, X. O., Batteur, S., Simon, R. P., and Greenberg, D.A. (2001). Neurogenesis in dentate subgranular zone and rostral subventricular zone after focal cerebral ischemia in the rat. Proc. Natl. Acad. Sci. U.S.A. 98, 4710-4715.

Kandasamy, M., Couillard-Despres, S., Raber, K. A., Stephan, M., Lehner, B., Winner, B., Kohl, Z., Rivera, F. J., Nguyen, H. P., Riess, O., Bogdahn, U., Winkler, J., von Horsten, S., and Aigner, L. (2010). Stem cell quiescence in the hippocampal neurogenic niche is associated with elevated transforming growth factor-beta signaling in an animal model of Huntington disease. J. Neuropathol. Exp. Neurol. 69, 717-728.

Karl, C., Couillard-Despres, S., Prang, P., Munding, M., Kilb, W., Brigadski, T., Plotz, S., Mages, W., Luhmann, H., Winkler, J., Bogdahn, U., and Aigner, L. (2005). Neuronal precursor-specific activity of a human doublecortin regulatory sequence. J. Neurochem. 92, 264-282.

Kaur, D., Rajagopalan, S., and Andersen, J. K. (2009). Chronic expression of $\mathrm{H}$-ferritin in dopaminergic midbrain neurons results in an age-related expansion of the labile iron pool and subsequent neurodegeneration: implications for Parkinson's disease. Brain Res. 1297, 17-22.

Kaur, D., Rajagopalan, S., Chinta, S., Kumar, J., Di Monte, D., Cherny, R. A., and Andersen, J. K. (2007). Chronic ferritin expression within murine dopaminergic midbrain neurons results in a progressive age-related neurodegeneration. Brain Res. 1140, 188-194.

Kempermann, G., Gast, D., Kronenberg, G., Yamaguchi, M., and Gage, F. H. (2003). Early determination and longterm persistence of adult-generated new neurons in the hippocampus of mice. Development 130, 391-399.

Kohl, Z., Kandasamy, M., Winner, B. Aigner, R., Gross, C., CouillardDespres, S., Bogdahn, U., Aigner, L., and Winkler, J. (2007). Physical activity fails to rescue hippocampal neurogenesis deficits in the R6/2 mouse model of Huntington's disease. Brain Res. 1155, 24-33.

Kustermann, E., Himmelreich, U., Kandal, K., Geelen, T., Ketkar,A., Wiedermann, D., Strecker, C., Esser, J., Arnhold, S., and Hoehn, M. (2008). Efficient stem cell labeling for MRI studies. Contrast Media Mol. Imaging 3, 27-37.

Ladewig, J., Koch, P., Endl, E., Meiners, B., Opitz, T., Couillard-Despres, S., Aigner, L., and Brustle, O. (2008). Lineage selection of functional and cryopreservable human embryonic stem cell-derived neurons. Stem Cells 26, 1705-1712.

Lazic, S. E., Grote, H., Armstrong, R. J. Blakemore, C., Hannan, A. J., van Dellen, A., and Barker, R. A. (2004). Decreased hippocampal cell proliferation in R6/1 Huntington's mice. Neuroreport 15, 811-813.

Le Bihan, D., Breton, E., Lallemand, D., Grenier, P., Cabanis, E., and LavalJeantet,M.(1986).MRimaging of intravoxel incoherent motions: application to diffusion and perfusion in neurologic disorders. Radiology 161, 401-407.

Lee, K. H., Byun, S. S., Paik, J. Y., Lee, S. Y., Song, S. H., Choe, Y. S., and Kim, B. T. (2003). Cell uptake and tissue distribution of radioiodine labelled D-luciferin: implications for luciferase based gene imaging. $\mathrm{Nucl}$. Med. Commun. 24, 1003-1009.

Liu, J., Solway, K., Messing, R. O., and Sharp, F. R. (1998). Increased neurogenesis in the dentate gyrus after transient global ischemia in gerbils. $J$. Neurosci. 18, 7768-7778.

Lois, C., and Alvarez-Buylla, A. (1994). Long-distance neuronal migration in the adult mammalian brain. Science 264, 1145-1148.

Ma, D. K., Bonaguidi, M. A., Ming, G. L., and Song, H. (2009). Adult neural stem cells in the mammalian central nervous system. Cell Res. 19, 672-682.

Magavi, S. S., Leavitt, B. R., and Macklis, J. D. (2000). Induction of neurogenesis in the neocortex of adult mice. Nature 405, 951-955.
Magnitsky, S., Watson, D. J., Walton, R. M., Pickup, S., Bulte, J. W., Wolfe, J. H., and Poptani, H. (2005). In vivo and ex vivo MRI detection of localized and disseminated neural stem cell grafts in the mouse brain. Neuroimage 26, 744-754.

Manganas, L. N., Zhang, X., Li, Y., Hazel, R. D., Smith, S. D., Wagshul, M. E., Henn, F., Benveniste, H., Djuric, P. M., Enikolopov, G., and Maletic-Savatic, M. (2007). Magnetic resonance spectroscopy identifies neural progenitor cells in the live human brain. Science 318, 980-985.

Markakis, E. A., and Gage, F. H. (1999). Adult-generated neurons in the dentate gyrus send axonal projections to field CA3 and are surrounded by synaptic vesicles. J. Comp. Neurol. 406, 449-460.

Marxreiter, F., Nuber, S., Kandasamy, M., Klucken, J., Aigner, R., Burgmayer, R., Couillard-Despres, S., Riess, O., Winkler, J., and Winner, B. (2009). Changes in adult olfactory bulb neurogenesis in mice expressing the $\mathrm{A} 30 \mathrm{P}$ mutant form of alpha-synuclein. Eur. J. Neurosci. 29, 879-890.

Modo, M., Hoehn, M., and Bulte, J. W. (2005). Cellular MR imaging. Mol. Imaging 4, 143-164.

Nieman, B. J., Shyu, J. Y., Rodriguez, J. J., Garcia, A. D., Joyner, A. L., and Turnbull, D. H. (2010). In vivo MRI of neural cell migration dynamics in the mouse brain. Neuroimage 50, 456-464.

Norman, A. B., Thomas, S. R., Pratt, R. G. Lu, S. Y., and Norgren, R. B. (1992). Magnetic resonance imaging of neural transplants in rat brain using a superparamagnetic contrast agent. Brain Res. 594, 279-283.

Panizzo, R. A., Kyrtatos, P. G., Price, A. N., Gadian, D. G., Ferretti, P., and Lythgoe, M. F. (2009). In vivo magnetic resonance imaging of endogenous neuroblasts labelled with a ferumoxide-polycation complex. Neuroimage 44, 1239-1246.

Parent, J. M., Vexler, Z. S., Gong, C., Derugin, N., and Ferriero, D. M. (2002). Rat forebrain neurogenesis and striatal neuron replacement after focal stroke. Ann. Neurol. 52, 802-813.

Parent, J. M., Yu, T. W., Leibowitz, R. T., Geschwind, D. H., Sloviter, R. S., and Lowenstein, D. H. (1997). Dentate granule cell neurogenesis is increased by seizures and contributes to aberrant network reorganization in the adult rat hippocampus. J. Neurosci. 17, 3727-3738.

Pfeuffer, J., Tkac, I., Provencher, S. W., and Gruetter, R. (1999). Toward an in vivo neurochemical profile: quantification of 18 metabolites in short-echo-time 
(1)H NMR spectra of the rat brain. J. Magn. Reson. 141, 104-120.

Pichler, A., Prior, J. L., and PiwnicaWorms, D. (2004). Imaging reversal of multidrug resistance in living mice with bioluminescence: MDR1 P-glycoprotein transports coelenterazine. Proc. Natl. Acad. Sci. U.S.A. 101, 1702-1707.

Ramm, P., Couillard-Despres, S., Plotz, S., Rivera, F. J., Krampert, M., Lehner, B., Kremer, W., Bogdahn, U., Kalbitzer, H. R., and Aigner, L. (2009). A nuclear magnetic resonance biomarker for neural progenitor cells: is it all neurogenesis? Stem Cells 27, 420-423.

Reumers, V., Deroose, C. M., Krylyshkina, O., Nuyts, J., Geraerts, M., Mortelmans, L., Gijsbers, R., Van den Haute, C., Debyser, Z., and Baekelandt, V. (2008). Noninvasive and quantitative monitoring of adult neuronal stem cell migration in mouse brain using bioluminescence imaging. Stem Cells 26, 2382-2390.

Reynolds, B. A., and Weiss, S. (1992). Generation of neurons and astrocytes from isolated cells of the adult mammalian central nervous system. Science 255, 1707-1710.

Rueger, M. A., Backes, H., Walberer, M., Neumaier, B., Ullrich, R., Simard, M. L., Emig, B., Fink, G. R., Hoehn, M., Graf, R., and Schroeter, M. (2010). Noninvasive imaging of endogenous neural stem cell mobilization in vivo using positron emission tomography. J. Neurosci. 30, 6454-6460.

Schafer, R. (2010). Labeling and imaging of stem cells - promises and concerns. Transfus. Med. Hemother. 37, 85-89.

Shaner, N. C., Campbell, R. E., Steinbach, P. A., Giepmans, B. N., Palmer, A. E., and Tsien, R. Y. (2004). Improved monomeric red, orange and yellow fluorescent proteins derived from Discosoma sp. red fluorescent protein. Nat. Biotechnol. 22, 1567-1572.

Shapiro, E. M., Gonzalez-Perez, O., GarciaVerdugo, J. M., Alvarez-Buylla, A., and Koretsky, A. P. (2006a). Magnetic resonance imaging of the migration of neuronal precursors generated in the adult rodent brain. Neuroimage 32, 1150-1157.

Shapiro, E. M., Sharer, K., Skrtic, S., and Koretsky, A. P. (2006b). In vivo detection of single cells by MRI. Magn. Reson. Med. 55, 242-249.

Slotkin, J. R., Cahill, K. S., Tharin, S. A., and Shapiro, E. M. (2007). Cellular magnetic resonance imaging: nanometer and micrometer size particles for noninvasive cell localization. Neurotherapeutics 4, 428-433.

Stanfield, B. B., and Trice, J. E. (1988). Evidence that granule cells generated in the dentate gyrus of adult rats extend axonal projections. Exp. Brain Res. 72, 399-406.

Sumner, J. P., Shapiro, E. M., Maric, D., Conroy, R., and Koretsky, A. P. (2009). In vivo labeling of adult neural progenitors for MRI with micron sized particles of iron oxide: quantification of labeled cell phenotype. Neuroimage 44, 671-678.

Toni, N., Laplagne, D. A., Zhao, C., Lombardi, G., Ribak, C. E., Gage, F.H., and Schinder, A. F. (2008). Neurons born in the adult dentate gyrus form functional synapses with target cells. Nat. Neurosci. 11, 901-907.

van Praag, H., Shubert, T., Zhao, C., and Gage, F. H. (2005). Exercise enhances learning and hippocampal neurogenesis in aged mice. J. Neurosci. 25, 8680-8685.

Vande Velde, G., Raman Rangarajan, J., Toelen, J., Dresselaers, T., Ibrahimi, A., Krylychkina, O., Vreys, R., Van der Linden, A., Maes, F., Debyser, Z., Himmelreich, U., and Baekelandt, V. (2011). Evaluation of the specificity and sensitivity of ferritin as an MRI reporter gene in mouse brain using lentiviral and adeno-associated viral vectors. Gene Ther. [Epub ahead of print].

Vreys, R., Soenen, S. J., De, C. M., and Van der Linden, A. (2011). Background migration of USPIO/MLs is a major drawback for in situ labeling of endogenous neural progenitor cells. Contrast Media Mol. Imaging 6, 1-6.

Vreys, R., Vande Velde, G., Krylychkina, O., Vellema, M., Verhoye, M. Timmermans, J. P., Baekelandt, V., and Van der Linden, A. (2010). MRI visualization of endogenous neural progenitor cell migration along the RMS in the adult mouse brain: validation of various MPIO labeling strategies. Neuroimage 49, 2094-2103.

Wen, P. H., Hof, P. R., Chen, X., Gluck, K. Austin, G., Younkin, S. G., Younkin, L. H., DeGasperi, R., Gama Sosa, M. A., Robakis, N. K., Haroutunian, V., and Elder, G. A. (2004). The presenilin-1 familial Alzheimer disease mutant P117L impairs neurogenesis in the hippocampus of adult mice. Exp. Neurol. 188, 224-237.

Winner, B., Cooper-Kuhn, C. M., Aigner, R., Winkler, J., and Kuhn, H. G. (2002). Long-term survival and cell death of newly generated neurons in the adult rat olfactory bulb. Eur. J. Neurosci. 16, 1681-1689.

Winner, B., Lie, D. C., Rockenstein, E., Aigner, R., Aigner, L., Masliah, E., Kuhn, H. G., and Winkler, J. (2004). Human wild-type alpha-synuclein impairs neurogenesis. J. Neuropathol. Exp. Neurol. 63, 1155-1166.

Winner, B., Rockenstein, E., Lie, D. C. Aigner, R., Mante, M., Bogdahn, U., Couillard-Despres, S., Masliah, E., and Winkler, J. (2008). Mutant alphasynuclein exacerbates age-related decrease of neurogenesis. Neurobiol. Aging 29, 913-925.

Yamaguchi, M., Saito, H., Suzuki, M., and Mori, K. (2000). Visualization of neurogenesis in the central nervous system using nestin promoter-GFP transgenic mice. Neuroreport 11, 1991-1996.

Yang, J., Liu, J., Niu, G., Chan, K. C., Wang, R., Liu, Y., and Wu, E. X. (2009). In vivo MRI of endogenous stem/pro- genitor cell migration from subventricular zone in normal and injured developing brains. Neuroimage 48, 319-328.

Zhang, R. L., Zhang, Z. G., Zhang, L., and Chopp, M. (2001). Proliferation and differentiation of progenitor cells in the cortex and the subventricular zone in the adult rat after focal cerebral ischemia. Neuroscience 105, 33-41.

Zhao, H., Doyle, T. C., Wong, R. J., Cao, Y., Stevenson, D. K., PiwnicaWorms, D., and Contag, C. H. (2004). Characterization of coelenterazine analogs for measurements of Renilla luciferase activity in live cells and living animals. Mol. Imaging 3, 43-54.

Zurkiya, O., Chan, A. W., and Hu, X. (2008). MagA is sufficient for producing magnetic nanoparticles in mammalian cells, making it an MRI reporter. Magn. Reson. Med. 59, 1225-1231.

Conflict of Interest Statement: The authors declare that the research was conducted in the absence of any commercial or financial relationships that could be construed as a potential conflict of interest.

Received: 25 January 2011; accepted: 27 April 2011; published online: 09 May 2011. Citation: Couillard-Despres S, Vreys R, Aigner L and Van der Linden A (2011) In vivo monitoring of adult neurogenesis in health and disease. Front. Neurosci. 5:67. doi: 10.3389/fnins.2011.00067

This article was submitted to Frontiers in Neurogenesis, a specialty of Frontiers in Neuroscience.

Copyright (c) 2011 Couillard-Despres, Vreys, Aigner and Van der Linden. This is an open-access article subject to a nonexclusive license between the authors and Frontiers Media SA, which permits use, distribution and reproduction in other forums, provided the original authors and source are credited and other Frontiers conditions are complied with. 